\title{
Nonlinear Control of a Novel Two-Link Pendulum
}

\author{
Laszlo Techy, C. Konda Reddy, Craig A. Woolsey, Chengyu Cao, and Naira Hovakimyan
}

\begin{abstract}
This paper describes nonlinear control of a two degree of freedom mechanical system which models a bird perched on a branch or cable. The primary contribution is the implementation, in a controlled laboratory experiment, of the recently developed $\mathcal{L}_{1}$ adaptive control approach. This technique, which allows fast adaptation with guaranteed stability margins, has been proposed for use in a variety of more sophisticated applications. Experimental results for this simple mechanical control system provide further motivation to pursue those applications. The system being considered is a variation of Spong's "Acrobot", a classic example of an underactuated mechanical control system. In addition to the $\mathcal{L}_{1}$ controller, a swing-up controller based on Spong's original work on collocated partial feedback linearization is implemented with switching logic.
\end{abstract}

\section{INTRODUCTION}

This paper describes modelling and control design for a mechanical system which resembles a bird perched on a branch or a cable. A primary function of the tail in birds is to provide flight stability [16], [17]. The tail may also provide a mechanism for active balance when a bird is perched [10]. In this capacity, the tail provides a counter-moment in response to any disturbance moments. As modeled in this paper, the system is similar to a planar double pendulum, modified to incorporate aerodynamic effects. As a mechanical system, the model is underactuated - it is assumed that the bird may use its tail muscles to exert a moment on its tail feathers, and the surrounding fluid, but that its feet can not exert any moment about the perch.

While the role of tail feathers in birds is of biological interest [10], the proposed model is also of interest to control designers as a variation of the now classic "Acrobot" described in [13]. As a simple mechanical control system, it exhibits some interesting features which challenge a number of existing nonlinear control methods. Since the system is underactuated, many standard nonlinear control techniques, such as backstepping or input-state linearization, are not directly applicable. The system structure is not amenable to the approach described in [11], nor may the structure be transformed into the desired form through feedback, as described in [15]. Energy shaping methods have been proposed for controlling underactuated mechanical systems [1], [18], but the feedback equivalence conditions can be difficult to

Laszlo Techy, Craig Woolsey, Chengyu Cao and Naira Hovakimyan are with the Nonlinear Systems Lab, Aerospace and Ocean Engineering, Virginia Tech, Blacksburg, VA 24061, USA. E-Mail: \{techy, cwoolsey, chengyu, nhovakim\}@vt.edu. Konda Reddy is with Control and Dynamical Systems, Caltech, Pasadena, CA 91125, USA. E-Mail: konda @cds.caltech.edu verify. Symmetries in the dynamics can simplify the analysis of feedback equivalence, but the system considered here exhibits no overall symmetry. Still, the dynamic structure is special in the sense that the kinetic energy depends only on the actuated variable and the potential energy depends only on the unactuated one. It may be that a simplified procedure for kinetic and potential energy shaping could be defined, as was done in [7] for a class of systems that includes the Acrobot example.

This paper is primarily concerned with experimentally demonstrating a new robust control approach, the " $\mathcal{L}_{1}$ adaptive control" technique [2], [3]. This new technique provides fast, online parameter adaptation for improved tracking with guaranteed stability margins. To bring the system state into a local neighborhood of the desired equilibrium, the energybased "collocated partial feedback linearization" method presented in [14] is also applied, with switching logic to shift to the $\mathcal{L}_{1}$ controller when appropriate. (With regard to the "perched bird" analogy, the authors are admittedly unaware of any fowl which are able, or so inclined, to swing themselves to an upright position.)

Section II describes the model of the system and Section III reviews both the $\mathcal{L}_{1}$ adaptive control method and the energy-based method used for swing-up. Section IV presents the results of simulations and experiments. Section V provides conclusions.

\section{DyNAMIC MODEL}

Consider a bird perched on a branch or a telephone line as depicted in Figure 1(a). It is assumed that the bird's feet exert no moment about the perch.

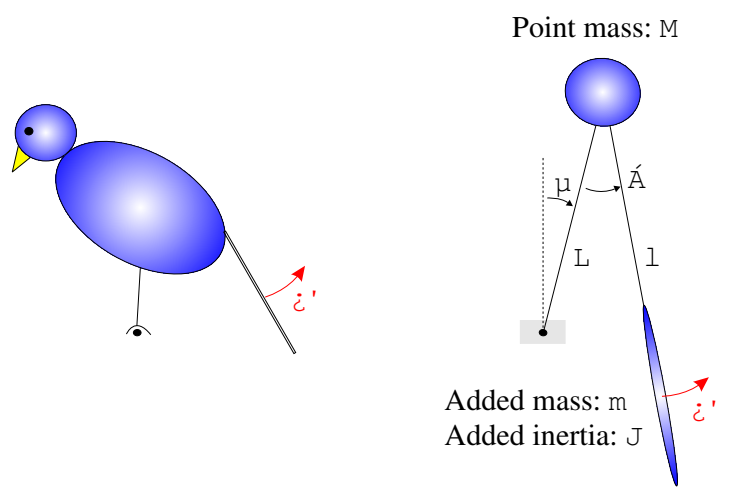

Fig. 1. (a) A bird perched on a wire (b) The two-link pendulum model of the perched bird. 
To move its tail through the surrounding air, the bird must exert a moment $\tau^{\prime}$ which results in a counter-moment that may help it to remain upright. In our model, we will assume that the air is quiescent; the bird must generate the stabilizing torque in still air. We model the bird as a simple inverted pendulum with an additional link as shown in Fig. 1(b). The inverted pendulum models the body and the massless legs whereas the additional link models the tail feathers. The body of the bird is modelled as a point mass $M$. The tail feathers are modelled as a massless flat plate. Though it has no actual mass, the plate induces added mass and added inertia, which account for the energy necessary to accelerate the air around the tail feathers as they move. Added mass is directional. While there is added mass in the direction normal to the flat plate, call it $m$, there is no added mass in tangential directions. We denote the added inertia of the disk about its center by $J$. To obtain an analytical expression for the added mass and added inertia of the tail feathers, we assume that the flat plate is actually an extremely oblate spheroid (i.e., a very thin pancake shape). In this case, according to [8],

$$
m \approx 5 \rho\left(\frac{4}{3} \pi r^{3}\right) \text { and } J \approx \frac{13 \rho}{2}\left(\frac{8}{15} \pi r^{5}\right),
$$

where $\rho$ is the density of the fluid and $r$ is the radius of the circular flat plate. The Lagrangian for this system is the kinetic energy minus the potential energy:

$$
L^{\prime}=\frac{1}{2}\left(\begin{array}{c}
\dot{\theta} \\
\dot{\phi}
\end{array}\right)^{\top}\left(\begin{array}{ll}
M_{11}^{\prime} & M_{12}^{\prime} \\
M_{21}^{\prime} & M_{22}^{\prime}
\end{array}\right)\left(\begin{array}{c}
\dot{\theta} \\
\dot{\phi}
\end{array}\right)-V^{\prime}(\theta, \phi)
$$

where

$$
\begin{aligned}
& M_{11}^{\prime}=M L^{2}+J+m(l-L \cos \phi)^{2}, \\
& M_{12}^{\prime}=M_{21}^{\prime}=-(J+m l(l-L \cos \phi)), \\
& M_{22}^{\prime}=J+m l^{2}, \\
& V^{\prime}(\theta, \phi)=-M g L(1-\cos \theta) .
\end{aligned}
$$

(The "prime" denotes a dimensional quantity; all terms are non-dimensionalized presently.) The second term in $M_{22}^{\prime}$ is the inertia due to the added mass of the disk about the point mass $M$. From equation (1), we get $J=\frac{13}{25} m r^{2}$. Thus, we have

$$
J+m l^{2}=m l^{2}\left(\frac{13}{25}\left(\frac{r}{l}\right)^{2}+1\right) .
$$

We will assume that $\frac{13}{25}\left(\frac{r}{l}\right)^{2} \ll 1$ so that the added inertia $J$ is negligible compared with the effect of added mass acting with a moment arm of length $l$. Define the following dimensionless parameters:

$$
\mu=\frac{m}{M}, \quad \lambda=\frac{l}{L}, \quad \text { and } \quad T=\sqrt{\frac{g}{L}} t .
$$

Then the nondimensional Lagrangian is

$$
L=\frac{1}{2}\left(\begin{array}{c}
\dot{\theta} \\
\dot{\phi}
\end{array}\right)^{\top}\left(\begin{array}{ll}
M_{11} & M_{12} \\
M_{21} & M_{22}
\end{array}\right)\left(\begin{array}{c}
\dot{\theta} \\
\dot{\phi}
\end{array}\right)-V(\theta, \phi)
$$

where overdots now (and in the rest of the paper) represent differentiation with respect to $T$ and where

$$
\begin{aligned}
M_{11}(\phi) & =1+\mu(\lambda-\cos \phi)^{2} \\
M_{12}(\phi) & =-\mu \lambda(\lambda-\cos \phi) \\
M_{22}(\phi) & =\mu \lambda^{2} \\
V(\theta) & =-(1-\cos \theta) .
\end{aligned}
$$

Ignoring all external moments except for the nondimensional input $\tau$ and the potential flow effect, which is accounted for in the kinetic energy, the Euler-Lagrange equations are

$$
\begin{aligned}
& \frac{d}{d t} \frac{\partial L}{\partial \dot{\theta}}-\frac{\partial L}{\partial \theta}=0 \\
& \frac{d}{d t} \frac{\partial L}{\partial \dot{\phi}}-\frac{\partial L}{\partial \phi}=\tau
\end{aligned}
$$

More explicitly, the equations of motion can be written

$$
\left(\begin{array}{ll}
M_{11} & M_{12} \\
M_{21} & M_{22}
\end{array}\right)\left(\begin{array}{l}
\ddot{\theta} \\
\ddot{\phi}
\end{array}\right)+\left(\begin{array}{ll}
C_{11} & C_{12} \\
C_{21} & C_{22}
\end{array}\right)\left(\begin{array}{c}
\dot{\theta} \\
\dot{\phi}
\end{array}\right)-\left(\begin{array}{c}
\sin \theta \\
0
\end{array}\right)=\left(\begin{array}{l}
0 \\
\tau
\end{array}\right),
$$

where the "Coriolis and centripetal" matrix elements are

$$
\begin{aligned}
& C_{11}(\phi, \dot{\theta}, \dot{\phi})=\mu(\lambda-\cos \phi) \sin \phi \dot{\phi}, \\
& C_{12}(\phi, \dot{\theta}, \dot{\phi})=\mu(\lambda-\cos \phi) \sin \phi \dot{\theta}-\mu \lambda \sin \phi \dot{\phi}, \\
& C_{21}(\phi, \dot{\theta}, \dot{\phi})=-\mu(\lambda-\cos \phi) \sin \phi \dot{\theta}, \\
& C_{22}(\phi, \dot{\theta}, \dot{\phi})=0 .
\end{aligned}
$$

\section{Control Design}

This section describes, in general terms, the $\mathcal{L}_{1}$ adaptive control method and the energy-based swing-up method. See the references for additional details.

\section{A. $\mathcal{L}_{1}$ Adaptive Control}

The most common control approach for nonlinear systems, when applicable, is to linearize the equations about an operating point or trajectory and control the system using a linear control method. Although the linear approximation is locally valid, there is typically model parameter uncertainty; the true state matrix differs from the one that is available to the control designer. The inherent robustness of linear control allows for some degree of model parameter uncertainty. Adaptive control provides additional robustness to parameter uncertainty.

The perched bird model clearly includes parameter uncertainty. The unsteady fluid effects are modelled, for example, in terms of inviscid potential flow using approximate values for added mass and added inertia. Because this experiment is intended to motivate more sophisticated applications of the $\mathcal{L}_{1}$ technique, we intentionally introduce additional parametric uncertainty.

A common adaptive control technique is Model Reference Adaptive Control (MRAC) in which the controller attempts to drive the true state to that of an ideal reference system despite parametric uncertainty [12]. In this approach, the 
control parameters are varied based on measurements. The rate at which these control parameters vary is defined by the adaptation gains which can, in principle, be chosen arbitrarily large to yield fast convergence. In reality, raising the adaptation gains leads to high-frequency control signals and a loss of robustness. Recently, Cao and Hovakimyan [2], [3] have developed a novel adaptive control method called $\mathcal{L}_{1}$ adaptive control. The $\mathcal{L}_{1}$ approach allows for fast parameter adaptation, providing asymptotic tracking of a reference input with a well-behaved control signal and guaranteed robustness margins [4], [5].

Consider the SISO, linear time-invariant system

$$
\begin{aligned}
\dot{\boldsymbol{x}}(t) & =\boldsymbol{A} \boldsymbol{x}(t)+\boldsymbol{B} u(t) \\
y(t) & =\boldsymbol{C}^{T} \boldsymbol{x}(t)
\end{aligned}
$$

where $\boldsymbol{A}$ contains uncertain parameters but $\boldsymbol{B}$ and $\boldsymbol{C}$ are known. (The $\mathcal{L}_{1}$ setting is actually more general; see [2].) Suppose that the uncertainty in $\boldsymbol{A}$ can be characterized by a vector of unknown constant parameters $\boldsymbol{\theta}$ as follows:

$$
\boldsymbol{A}=\boldsymbol{A}_{0}-\boldsymbol{B} \boldsymbol{\theta}^{T} \text {. }
$$

In this case, the standard matching assumptions from direct MRAC theory are automatically satisfied. Although $\boldsymbol{\theta}$ is unknown, its value is assumed to lie in a known compact set $\Theta$.

Partition the control input $u(t)$ into a linear and an adaptive part $u(t)=u_{l}(t)+u_{a}(t)$. The linear controller can be designed for the nominal system (defined by $\boldsymbol{A}_{0}$ ) using LQR theory, for example, to obtain a closed-loop Hurwitz state matrix $\boldsymbol{A}_{m}=\boldsymbol{A}_{0}-\boldsymbol{B} \boldsymbol{K}^{T}$.

Define the following state predictor:

$$
\begin{aligned}
\dot{\hat{\boldsymbol{x}}}(t) & =\boldsymbol{A}_{m} \hat{\boldsymbol{x}}(t)+\boldsymbol{B}\left(u_{a}(t)-\hat{\boldsymbol{\theta}}^{T}(t) \boldsymbol{x}(t)\right) \\
\hat{y}(t) & =\boldsymbol{C}^{T} \hat{\boldsymbol{x}}(t),
\end{aligned}
$$

where $\hat{\boldsymbol{x}}(t), \hat{y}(t)$ and $\hat{\boldsymbol{\theta}}(t)$ are estimates of $\boldsymbol{x}(t), y(t)$ and $\boldsymbol{\theta}$, respectively. The control objective is to make the system output $y(t)$ track an arbitrary, smooth reference signal $r(t)$.

Consider the adaptive control law

$$
u_{a}(s)=C_{f}(s)\left(\bar{r}(s)+k_{g} r(s)\right)
$$

where $C_{f}(s)$ is a low-pass filter with unity DC gain and the constant $k_{g}$ is a feed-forward gain that ensures asymptotic tracking for step input. (All signals in (4) are expressed in the Laplace domain.) The signal $\bar{r}(s)$ is the Laplace transform of

$$
\bar{r}(t)=\hat{\boldsymbol{\theta}}^{T}(t) \boldsymbol{x}(t)
$$

The $\mathcal{L}_{1}$ adaptive controller (4) reduces MRAC when $C_{f}(s)=1$. The adaptation law is

$$
\dot{\hat{\boldsymbol{\theta}}}(t)=\boldsymbol{\Gamma}_{x} \operatorname{Proj}\left(\boldsymbol{x}(t) \tilde{\boldsymbol{x}}^{T}(t) \boldsymbol{P} \boldsymbol{B}, \hat{\boldsymbol{\theta}}(t)\right),
$$

where $\boldsymbol{\Gamma}_{x}$ term is the matrix of adaptive gains and $\operatorname{Proj}(\boldsymbol{y}, \hat{\boldsymbol{\theta}}(t))$ is the projection operator [9], which ensures that the adaptive parameters remain bounded. In the argument of the projection operator, $\tilde{\boldsymbol{x}}(t)=\hat{\boldsymbol{x}}(t)-\boldsymbol{x}(t)$ is the state error and $\boldsymbol{P}$ is the symmetric, positive definite solution of the Lyapunov equation

$$
\boldsymbol{A}_{m}^{T} \boldsymbol{P}+\boldsymbol{P} \boldsymbol{A}_{m}=-\boldsymbol{Q},
$$

where $Q$ is any symmetric, positive definite matrix.

To summarize, the adaptive component $u_{a}$ is given in (4) and (5), along with the predictor dynamics (3) and the adaptation law (6).

Define the positive definite Lyapunov-function candidate

$$
V(\tilde{\boldsymbol{x}}, \tilde{\boldsymbol{\theta}})=\tilde{\boldsymbol{x}}^{T} \boldsymbol{P} \tilde{\boldsymbol{x}}+\tilde{\boldsymbol{\theta}}^{T} \boldsymbol{\Gamma}_{x}^{-1} \tilde{\boldsymbol{\theta}},
$$

where $\tilde{\boldsymbol{\theta}}=\hat{\boldsymbol{\theta}}-\boldsymbol{\theta}$ is the estimation error. It is straight forward to show that

$$
\dot{V}(\tilde{\boldsymbol{x}}, \tilde{\boldsymbol{\theta}}) \leq-\tilde{\boldsymbol{x}}^{T} \boldsymbol{Q} \tilde{\boldsymbol{x}} \leq 0 .
$$

This suggests that the error dynamics converge, however one cannot deduce stability from this result directly. It must also be shown that the predictor state remains bounded. The boundedness of the predictor state and asymptotic convergence of the error to zero are shown in [2] provided one additional condition is satisfied - the low-pass filter $C_{f}(s)$ must be constructed to meet the $\mathcal{L}_{1}$ stability criterion:

$$
\|\overline{\boldsymbol{G}}(s)\|_{\mathcal{L}_{1}} \theta_{\max } \leq 1
$$

where $\|\overline{\boldsymbol{G}}(s)\|_{\mathcal{L}_{1}}$ is the $\mathcal{L}_{1}$ gain of

$$
\overline{\boldsymbol{G}}(s)=\left(s \boldsymbol{I}-\boldsymbol{A}_{m}\right)^{-1} \boldsymbol{B}\left(C_{f}(s)-1\right),
$$

where

$$
\theta_{\max }=\max _{\boldsymbol{\theta} \in \boldsymbol{\Theta}} \sum_{i=1}^{n}\left|\theta_{i}\right| .
$$

The following result follows from the results in [2].

Proposition 3.1: Assume that the $\mathcal{L}_{1}$ stability criterion (7) holds. Then the control law (4) together with adaptation law (6) and predictor (3) ensures that $\lim _{t \rightarrow \infty} \tilde{\boldsymbol{x}}(t)=\mathbf{0}$.

\section{B. Collocated Partial Feedback Linearization}

Although underactuated systems are generally not inputstate linearizable, Spong [13] applied a partial linearization method called collocated partial feedback linearization to swing up the Acrobot. Consider the class of two degree of freedom (DOF) underactuated mechanical systems described by the equations ${ }^{1}$

$$
\begin{aligned}
& M_{11} \ddot{q}_{1}+M_{12} \ddot{q}_{2}+C_{1}+G_{1}=0, \\
& M_{21} \ddot{q}_{1}+M_{22} \ddot{q}_{2}+C_{2}+G_{2}=\tau,
\end{aligned}
$$

where $q_{1}$ and $q_{2}$ are the generalized coordinates. The terms $C_{i}$ and $G_{i}$ represent Coriolis-centripetal and gravity effects,

\footnotetext{
${ }^{1}$ Though we only consider a two-DOF system, any underactuated $n$-DOF system can be be partitioned in this lower underactuated form.
} 
respectively. (To simplify notation, the dependence of $M_{i j}$, $C_{i}$, and $G_{i}$ on the state is not indicated explicitly.) The class of systems (8-9) describes the perched bird model described in Section II, with $\left(q_{1}, q_{2}\right)=(\theta, \phi)$ and $G_{2}=0$. Since the generalized inertia matrix is positive definite, $M_{11} \neq 0$ and equation (8) can be rewritten as

$$
\ddot{q}_{1}=-M_{11}^{-1}\left(M_{12} \ddot{q}_{2}+C_{1}+G_{1}\right) \text {. }
$$

Substituting (10) into (9),

$$
\tilde{M}_{22} \ddot{q}_{2}+\tilde{C}_{2}+\tilde{G}_{2}=\tau,
$$

where

$$
\begin{aligned}
\tilde{M}_{22} & =M_{22}-M_{21} M_{11}^{-1} M_{12}, \\
\tilde{C}_{2} & =C_{2}-M_{21} M_{11}^{-1} C_{1}, \\
\tilde{G}_{2} & =G_{2}-M_{21} M_{11}^{-1} G_{1} .
\end{aligned}
$$

By defining the input

$$
\tau=\tilde{M}_{22} u+\tilde{C}_{2}+\tilde{G}_{2}
$$

the equations of motion (8-9) become

$$
\begin{gathered}
M_{11} \ddot{q}_{1}+C_{1}+G_{1}=-M_{12} u, \\
\ddot{q}_{2}=u,
\end{gathered}
$$

where the directly actuated subsystem has been rendered linear. Considering only the $q_{2}$ subsystem, for the moment, any linear control design technique may be applied to drive $q_{2}$ to zero. For example, choosing static state feedback

$$
u=-K_{1} q_{2}-K_{2} \dot{q}_{2}+\bar{u}
$$

with $K_{1}>0$ and $K_{2}>0$ and $\bar{u}=0$ stabilizes the $q_{2}$ subsystem.

The essential problem is to choose $\bar{u}$ to drive the complete system to a desired state. In the similar setting of inputoutput linearization, one investigates the zero-dynamics to ensure that they are asymptotically stable. For collocated partial feedback linearization, the zero-dynamics are often unstable and choosing $\bar{u}$ appropriately is a nontrivial task. We use an energy based technique, similar to the one used to stabilize the Acrobot in [14], to bring the system state within the region of attraction of the locally stabilizing controller discussed in Section III-A.

To perform the swing-up maneuver, the controller must increase the total energy of the system

$$
E=\frac{1}{2}\left(\begin{array}{c}
\dot{q}_{1} \\
\dot{q}_{2}
\end{array}\right)^{T}\left(\begin{array}{ll}
M_{11} & M_{12} \\
M_{21} & M_{22}
\end{array}\right)\left(\begin{array}{c}
\dot{q}_{1} \\
\dot{q}_{2}
\end{array}\right)+V\left(q_{1}, q_{2}\right)
$$

¿from its minimum value. Let $E_{\text {eq }}$ represent the energy of the system at the desired equilibrium. Let $\bar{u}=K_{3} \dot{q}_{1}\left(E-E_{\text {eq }}\right)=$ $K_{3} \dot{q}_{1} \tilde{E}$ so that

$$
u=-K_{1} q_{2}-K_{2} \dot{q}_{2}+K_{3} \dot{q}_{1} \tilde{E} .
$$

With appropriately chosen gains, this approach provides convergence of the system state to a constant-energy level set [14]. When the trajectory passes through the region of attraction of the $\mathcal{L}_{1}$ local controller, the control law is switched to asymptotically stabilize the desired equilibrium. Specifically, the switch occurs at the boundary of the compact set $\Omega_{c}=\left\{\boldsymbol{x}|| E-E_{\text {eq }} \mid \leq \epsilon\right\}$ where $\epsilon$ may be computed from Lyapunov analysis or from experiments. If the system can be shown to switch only once, a dwell-time condition is satisfied which ensures asymptotic convergence to the desired equilibrium [6].

\section{RESUlts FROM Simulation AND EXPERIMENT}

Section IV-A describes simulations using the local adaptive controller while Section IV-B incorporates the energy based swing-up controller, as well. Section IV-C discusses an experimental application.

\section{A. Local $\mathcal{L}_{1}$ Adaptive Control}

Because the objective is stabilization (regulation), the feedforward term $k_{g} r(s)$ in (4) may be disregarded. The equations of motion, linearized about the origin, are

$$
\boldsymbol{A}_{0}=\left(\begin{array}{cccc}
0 & 0 & 1 & 0 \\
0 & 0 & 0 & 1 \\
1 & 0 & 0 & 0 \\
\frac{\lambda-1}{\lambda} & 0 & 0 & 0
\end{array}\right), \quad \boldsymbol{B}=\left(\begin{array}{c}
0 \\
0 \\
\frac{\lambda-1}{\lambda} \\
\left(\frac{\lambda-1}{\lambda}\right)^{2}+\frac{1}{\mu \lambda^{2}}
\end{array}\right)
$$

For the simulation, as well as the experimental apparatus discussed in Section IV-C, the nominal parameter values are

$$
\lambda=4 \quad \text { and } \quad \mu=0.211 \text {. }
$$

An LQR controller was designed using the state and control penalty matrices

$$
\boldsymbol{Q}_{L Q R}=\left(\begin{array}{cccc}
100 & 0 & 0 & 0 \\
0 & 10 & 0 & 0 \\
0 & 0 & 1 & 0 \\
0 & 0 & 0 & 1
\end{array}\right), \quad R_{L Q R}=1000
$$

In addition, an $\mathcal{L}_{1}$ controller was designed with the parameter adaptation gain matrix $\boldsymbol{\Gamma}_{x}=30000 \cdot \boldsymbol{I}$ and a simple first-order low-pass filter

$$
C_{f}(s)=\frac{\omega_{c}}{s+\omega_{c}} .
$$

Recall that the filter bandwidth $\omega_{c}$ must be chosen such that the system satisfies the $\mathcal{L}_{1}$ stability criterion (7). The $\mathcal{L}_{1}$ gain of $\overline{\boldsymbol{G}}(s)$ may be computed numerically for different choices of $\omega_{c}$. In order to illustrate the effectiveness of the $\mathcal{L}_{1}$ controller, the known state matrix was perturbed according to (2) with $\boldsymbol{\theta}^{T}=-0.45\left[\begin{array}{llll}1 & 1 & 1 & 1\end{array}\right]^{T}$ and $\theta_{\max }$ was chosen larger than $\|\boldsymbol{\theta}\|_{1}$. (This is a rather large perturbation, given that the equations of motion are nondimensional.) Checking condition (7), one finds that $\omega_{c}=3$ is acceptable.

The initial state for simulations was $[\theta, \phi, \dot{\theta}, \dot{\phi}]=$ $[0.1,0.1,0,0]$. Three cases were compared: (1) the LQR controller applied to the nominal system with state matrix 

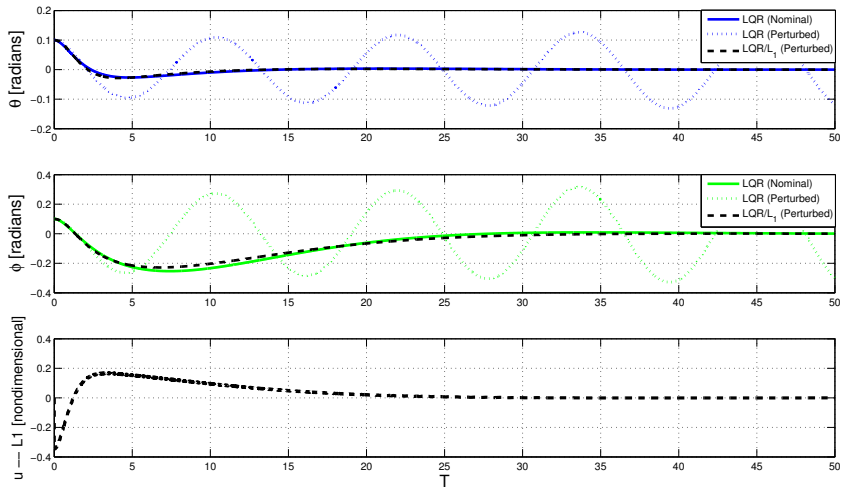

Fig. 2. Simulation results for the $\mathcal{L}_{1}$ controller.

$\boldsymbol{A}_{0}$, (2) the LQR controller applied to the perturbed system with state matrix $\boldsymbol{A}=\boldsymbol{A}_{0}-\boldsymbol{B} \boldsymbol{\theta}^{T}$, and (3) the LQR/L $\mathcal{L}_{1}$ controller applied to the perturbed system. The results are shown in Figure 2. In the figure, it is clear that the $\mathcal{L}_{1}$ controller yields much better performance than the LQR controller with the given model uncertainties. In fact, for a similar uncertainty with slightly larger magnitude, the LQR controller fails entirely to stabilize the system, while the $\mathcal{L}_{1}$ controller's performance is almost indistinguishable from the LQR controller's performance for the nominal system. The control signal for the $\mathcal{L}_{1}$ controller is also shown in Figure 2 to illustrate that there are no high frequency oscillations, as one might otherwise expect with such a large adaptation gain.

\section{B. Energy Based Swing-up Control}

To bring the system into the region of attraction of the $\mathrm{LQR} / \mathcal{L}_{1}$ controller, return to the original system equations and define $\tau$ according to (12). Specifically, let

$$
\begin{aligned}
\tau= & \frac{1}{1+\mu(\lambda-\cos \phi)^{2}}\left[\left(2 \mu^{2} \lambda(\lambda-\cos \phi)^{2} \sin \phi \dot{\theta} \dot{\phi}\right)\right. \\
& -\mu(\lambda-\cos \phi)\left(1+\mu(\lambda-\cos \phi)^{2}\right) \sin \phi \dot{\theta}^{2} \\
& \left.-\mu \lambda(\lambda-\cos \phi)\left(\sin \theta+\mu \lambda \sin \phi \dot{\phi}^{2}\right)+u \lambda^{2} \mu\right] .
\end{aligned}
$$

Then the original system is transformed through feedback into the system described by equations (13), (14):

$$
\begin{aligned}
\ddot{\theta}= & \frac{1}{1+\mu(\lambda-\cos \phi)^{2}}(\mu \lambda(\lambda-\cos \phi) u+\sin \theta \\
& \left.-2 \mu(\lambda-\cos \phi) \sin \phi \dot{\theta} \dot{\phi}+\lambda \mu \sin \phi \dot{\phi}^{2}\right) \\
\ddot{\phi}= & u .
\end{aligned}
$$

Now, define $u$ according to (15). Including an actuator torque magnitude limit in the model, the following control gains were derived through iterative simulation:

$$
K_{1}=0.1, \quad K_{2}=0.1, \quad \text { and } \quad K_{3}=-1 .
$$
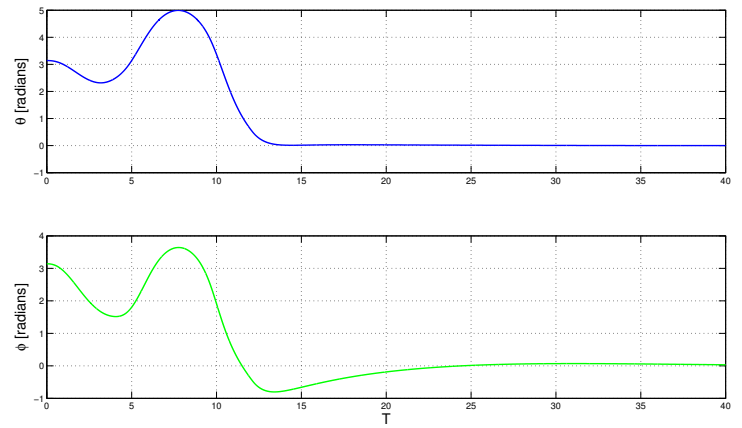

Fig. 3. Simulation results for the swing-up/ $\mathcal{L}_{1}$ controller.

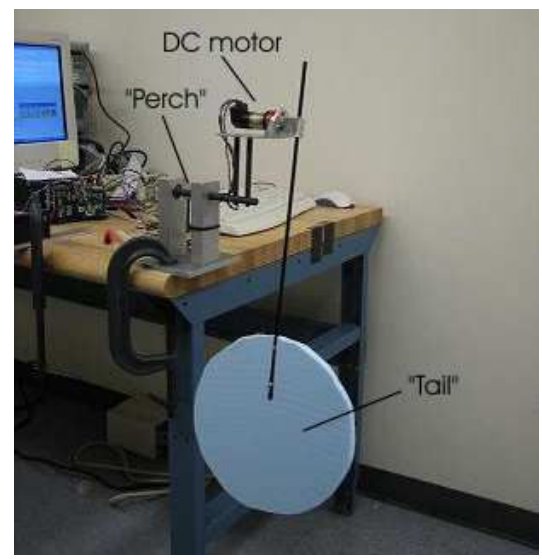

Fig. 4. Photo of the experimental apparatus.

Starting from the downward-hanging equilibrium point corresponding to $(\theta, \phi)=(\pi, \pi)$, the energy based controller discussed in Section III-B brings the system within a compact set $\Omega_{c}$ as discussed at the end of that section. The system then switches to the local $\mathcal{L}_{1}$ adaptive controller, as presented in Section IV-A. At the instant the switch occurs, the predictor is initialized with the current system states and the adaptive parameters are set to zero. The results, shown in Figure 3, clearly illustrate convergence to the upright equilibrium.

\section{Experimental Results}

An experimental implementation of the system considered in this paper is shown in Figure 4. The "body" is a DC motor that drives the "tail," a hollow carbon-fiber rod with a large foam disk. The experiment is controlled by a PC running real-time code with a sampling frequency of $5 \mathrm{kHz}$. This high sampling frequency is necessary in order to implement a large adaptation gain for fast adaptation. In experiments, both the swing-up and $\mathcal{L}_{1}$ controllers provided good performance, as indicated in Figures 5 and 6.

\section{CONCLUSions}

The recently developed $\mathcal{L}_{1}$ adaptive control algorithm was implemented experimentally for a novel underactuated 

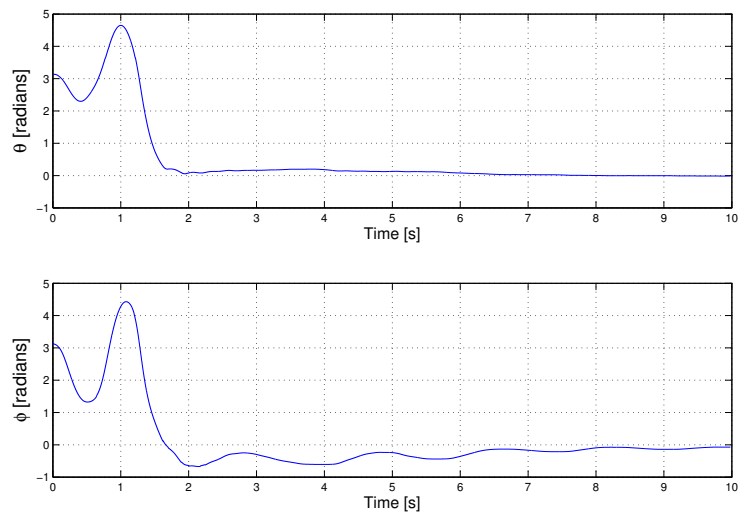

Fig. 5. Experimental results for the swing-up/ $\mathcal{L}_{1}$ controller.

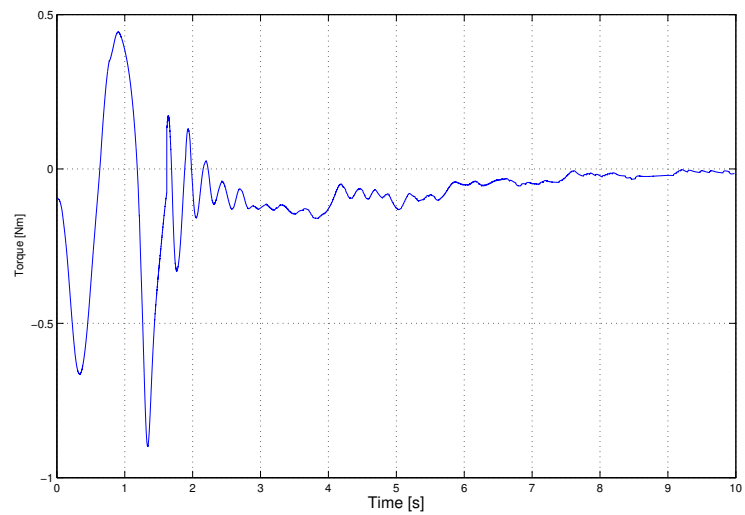

Fig. 6. Time history of the control input.

mechanical system inspired by a bird perched on a branch or a cable. Recognizing the similarity between the system considered here and the classic Acrobot, the local adaptive controller was combined with a swing-up controller that was originally proposed for the Acrobot. An energy-based switching criterion was used to transfer the system from the swing-up controller to the local $\mathcal{L}_{1}$ adaptive controller. In simulation, the controller exhibited excellent performance in the face of large model parameter uncertainty. The controller also performed quite well when implemented in a benchtop control experiment. The experimental performance of the $\mathcal{L}_{1}$ adaptive controller, in particular, further recommends its application in more sophisticated problems of greater engineering significance.

Acknowledgements. The authors gratefully acknowledge the input of the anonymous reviewers. The work was sponsored in part by NSF Grant \#CMS0133210 and AFOSR Grant \#FA9550-05-1-0157.

\section{REFERENCES}

[1] G. Blankenstein, R. Ortega, and A. J. van der Schaft. The matching conditions of controlled Lagrangians and IDA-Passivity Based Control. International Journal of Control, 75(9):645-665, 2002.

[2] C. Cao and N. Hovakimyan. Design and analysis of a novel L1 adaptive controller, Part I: Control signal and asymptotic stability. In Proc. American Control Conference, pages 3397-3402, 2006.

[3] C. Cao and N. Hovakimyan. Design and analysis of a novel L1 adaptive controller, Part II: Guaranteed transient performance. In Proc. American Control Conference, pages 3402-3408, 2006.

[4] C. Cao and N. Hovakimyan. Guaranteed transient performance with $\mathcal{L}_{1}$ adaptive controller for systems with unknown time-varying parameters: Part I. In Proc. of American Control Conf., New York, NY, 2007.

[5] C. Cao and N. Hovakimyan. Stability margins of $\mathcal{L}_{1}$ adaptive controller: Part II. In Proc. of American Control Conf., New York, NY, 2007.

[6] D. Liberzon. Switching in Systems and Control. Birkhauser, Boston, 2003.

[7] A. D. Mahindrakar, A. Astolfi, R. Ortega, and G. Viola. Further constructive results on interconnection and damping assignment control of mechanical systems: The Acrobot example. In Proc. American Control Conference, pages 5584-5589, 2006.

[8] J. N. Newman. Marine Hydrodynamics. The MIT Press, Cambridge, MA, 1977.

[9] J. B. Pomet and L. Praly. Adaptive nonlinear regulation: Estimation from the Lyapunov equation. IEEE Transactions on Automatic Control, 37(6):729-740, 1992.

[10] M. Romera-Pujante, H. Hoi, and D. Blomqvist. The importance of tail length for habitat use in Bearded Tit: An experimental study. Ibis, 147:464-470, 2005.

[11] D. Seto and J. Baillieul. Control problems in super-articulated mechanical systems. IEEE Transactions on Automatic Control, 39(12):2442 $-2453,1994$

[12] J. E. Slotine and W. Li. Applied Nonlinear Control. Prentice Hall, 1991.

[13] M. Spong. The swing-up control problem for the Acrobot. IEEE Control Systems Magazine, 15(1):49-55, 1995.

[14] M. Spong. Energy based control of a class of underactuated mechanical system. In Proc. 13th IFAC World Congress, volume F, pages 431 $-436,1996$.

[15] Z. Sun, S. S. Ge, and T. H. Lee. Nonregular backstepping design of underactuated mechanical systems. In Proc. 2001 IEEE International Conference on Control Applications, pages 173 - 178, 2001.

[16] G. K. Taylor and A. L. R. Thomas. Animal flight dynamics II: Longitudinal stability in flapping flight. Journal of Theoretical Biology, 214:351-370, 2002.

[17] A. L. R. Thomas and G. K. Taylor. Animal flight dynamics I: Stability in gliding flight. Journal of Theoretical Biology, 212:399-424, 2001.

[18] C. Woolsey, C. K. Reddy, A. M. Bloch, D. E. Chang, N. E. Leonard, and J. E. Marsden. Controlled Lagrangian systems with gyroscopic forcing and dissipation. European Journal of Control, 10(5):478-496, 2004. 